\title{
FUNCTIONS ON LOCALLY COMPACT GROUPS
}

\author{
GEORGE W. MACKEY ${ }^{1}$
}

\section{INTRODUCTION}

1. Background. The subject of this address is a branch of mathematics which may be regarded as a combination of the classical theory of representations of finite groups by matrices and that part of analysis centering around the theory of Fourier series and integrals. The connection between these two apparently diverse subjects arises simply enough from the fact that the real numbers and the real numbers modulo $2 \pi$ form groups under addition.

In one form of the theory of representations of a finite group $G$ a central role is played by the so-called group ring or group algebra. This is usually defined as the set of all formal linear combinations of group elements $c_{1} s_{1}+c_{2} s_{2}+\cdots+c_{n} s_{n}$, where each $s_{i} \in G$ and each $c_{i}$ is a complex number. Two such expressions are added in the obvious manner and are multiplied by writing down the formal product and simplifying by means of the distributive law and the given multiplication of group elements. It may also be defined (and this is the definition we shall use) as the vector space of all complex-valued functions on $G$ with multiplication defined by the formula

$$
(f * g)(s)=\sum_{t \in G} f\left(s t^{-1}\right) g(t) .
$$

If we regard $c_{1} s_{1}+c_{2} s_{2}+\cdots+c_{n} s_{n}$ as the function whose value at $s_{i}$ is $c_{i}$, it is not difficult to see that these two definitions amount to the same thing. A basic result in the theory of group representations asserts that the group algebra is a direct sum of minimal two-sided ideals. Now it is easy to see that a linear subspace $M$ of the group algebra is a left (right) ideal if and only if it is closed with respect to left (right) translation; that is, if and only if $f \in M$ implies that $f_{a}\left(f_{a}\right)$ is in $M$ for all $a$ in $G$ where $a f(s)=f(a s)$ and $f_{a}(s)=f(s a)$. Thus every function on $G$ is uniquely expressible as a sum of functions each of which has the property that its right and left translates generate a linear subspace which is minimal with respect to translation invariance.

An address delivered before the Philadelphia meeting of the Society on April 29, 1949, by invitation of the Committee to Select Hour Speakers for Eastern Sectional Meetings; received by the editors October 3, 1949.

1 The author is a fellow of the John Simon Guggenheim Memorial Foundation. 
On the other hand, Fourier's theorem asserts that every member of a large class of functions on the line with period $2 \pi$ (that is, functions on the group of the reals modulo $2 \pi$ ) may be written in the form

$$
\sum_{n=-\infty}^{\infty} c_{n} \exp (i n x)
$$

where the $c_{n}$ are complex numbers, $n$ is an integer, and $i^{2}=-1$. Here the functions $\exp (i n x)$ generate one-dimensional and hence minimal subspaces which are certainly translation invariant.

The analogy is clear. In each case there is a theorem asserting that a fairly general function on a group is a sum of functions having especially simple translation properties. It is natural to seek general theorems about the translation properties of functions on groups which include these as special cases. In this address I shall describe some of the work that has been done in this direction in the last twenty years or so and indicate its connections with other branches of mathematics.

There will be no attempt at completeness of coverage except from the special point of view here adopted. Many topics more or less closely related will not be discussed at all. In particular the emphasis will be on theorems valid for quite general groups and on the papers in the literature where these theorems are established in general. Thus in spite of his pioneering work in the harmonic analysis of functions on the line, there will be little mention of the name of Norbert Wiener.

In gathering this material together I have benefited greatly from conversations with I. Kaplansky, L. H. Loomis, F. I. Mautner, and I. E. Segal, and I wish to take this opportunity to acknowledge my indebtedness to them.

2. Preliminaries. In the theory of Fourier series and integrals the topology of the underlying group plays a role. Accordingly it is natural to consider topological groups; that is, groups which are at the same time topological spaces in such a manner that the group operations are continuous. Since every group is a topological group with respect to the topology under which every set is closed, discrete and in particular finite groups are not excluded. Among topological groups, those admitting a compact neighborhood of the identity, the so-called locally compact groups, present themselves as especially suitable for study. According to a refinement of an important 
theorem of A. Haar [34], ${ }^{2}$ every such group ${ }^{3}$ admits an essentially unique Borel measure ${ }^{4}$ which is invariant under right (left) translation. When $G$ is the real line, this measure reduces to ordinary Lebesgue measure. In the general case its existence makes it possible to extend most of the methods, notions, and results of the theory of Lebesgue integration to the study of functions on arbitrary locally compact groups. In particular one may speak of the class $\mathcal{L}^{1}(G)$ of functions summable on the group of the class $\mathcal{L}^{2}(G)$ of functions of summable square, of the integral $\int f(x) d x$ of a function in $\mathcal{L}^{1}(G)$, and so on.

It is only when $G$ is finite that a satisfactory theory of the set of all functions on $G$ can be given. In general it seems to be necessary to restrict attention to a large but not all inclusive subfamily. This family is usually taken to be the set of all functions on the group having some simple topological or measure theoretic property. It is ordinarily linear and translation invariant and provided with a natural topology. The problem ${ }^{5}$ is then to give as complete an analysis as possible of the structure of the set of closed translation invariant linear subspaces. Of the families of functions one might consider, two have properties which make them especially suitable for such a study and have received a great deal of attention. These are the two measure theoretically defined families mentioned in the preceding paragraph, $\mathcal{L}^{1}(G)$ and $\mathcal{L}^{2}(G)$. The first may be made into an algebra which is a generalization of the group algebra of a finite group, multiplication being defined by the formula

$$
f * g(s)=\int f\left(s t^{-1}\right) g(t) d t
$$

where integration is with respect to right invariant Haar measure. Whenever $f$ and $g$ are in $\mathcal{L}^{1}(G)$, the right-hand side exists for almost all $s$ and defines a function in $\mathcal{L}^{1}(G)$. It is not difficult to show that a linear subspace of $\mathcal{L}^{1}(G)$ closed in the usual norm is right (left) invariant under translation if and only if it is a right (left) ideal in the group algebra. In this case then the general problem becomes that of

\footnotetext{
${ }^{2}$ Numbers in brackets refer to the bibliography at the end of the paper.

${ }^{3}$ Haar considered only separable groups and did not prove uniqueness. Uniqueness was first proved by von Neumann [55] and the extension to the nonseparable case was first carried out by Weil [77]. Cf. also von Neumann [56] and Kakutani [38].

${ }^{4}$ In the terminology of Halmos [36].

${ }^{5}$ Cf. $[66$, p. 865$]$.
} 
studying the ideal theory of a certain Banach algebra. $\mathcal{L}^{2}(G)$ on the other hand, while not in general an algebra, is a Hilbert space and possesses the especially simple structure of such spaces. In particular the possibility of taking orthogonal complements makes its particularly suitable for direct sum-like decompositions.

\section{Compact Groups}

3. Two-sided invariant subspaces and the Peter-Weyl theorem. When the group in question is not only locally compact but compact, a rather complete extension of the theory for finite groups may be given. This theory was first developed by F. Peter and H. Weyl [60] in 1927. These authors restricted themselves to Lie groups, but only because the general measure of Haar, which incidentally for compact groups is always both left and right invariant, was not then available. Their theory goes over to the general case without change. ${ }^{6}$ The fundamental result of this theory-the celebrated Peter-Weyl theorem-may be stated in the following form ${ }^{7}: \mathcal{L}^{2}(G)$ is the direct sum of finite-dimensional minimal two-sided invariant subspaces $\mathfrak{J}_{\alpha}$. Each consists entirely of continuous functions. Every continuous function on $G$ is a uniform limit of finite sums of functions from the $J_{\alpha}$. The theory of the closed two-sided invariant subspaces of $\mathcal{L}^{2}(G)$ now follows at once. Every such subspace is the direct sum of the $J_{\alpha}$ 's which it contains and every family of $J_{\alpha}$ 's defines a unique such subspace. In particular there is a natural inclusion preserving correspondence between the subfamilies of the family of all $J_{\alpha}$ 's and the closed two-sided invariant subspaces of $\mathcal{L}^{2}(G)$. The theory of $\mathcal{L}^{1}(G)$ is hardly less simple. Each $\nearrow_{\alpha}$ is a minimal two-sided ideal and every closed two-sided ideal is the closed linear span of the $J_{\alpha}$ 's which it contains. For compact groups there is a natural one-to-one correspondence between the closed invariant subspaces of $\mathcal{L}^{2}(G)$ on the one hand and those of $\mathcal{L}^{1}(G)$ on the other.

4. One-sided invariant subspaces and representations. Every closed right invariant subspace $R$ of $\mathcal{L}^{2}(G)$ or $\mathcal{L}^{1}(G)$ is the closed linear span of the set of intersections $R \cap J_{\alpha}$. Thus the analysis of the right invariant subspaces of $\mathcal{L}^{2}(G)$ or $\mathcal{L}^{1}(G)$ reduces to that of the $J_{\alpha}$. Now it is a well known theorem of Wedderburn that each $\Im_{\alpha}$, being simple as an algebra, is isomorphic to the algebra of all linear transformations of an $n$-dimensional vector space into itself. Here $n=n_{\alpha}$ is a

${ }^{6}$ As far as separable groups are concerned this was pointed out by Haar himself as a corollary to the existence of his measure.

${ }^{7}$ Cf. Köthe [42]. 
positive integer depending on $\alpha$. It follows that each $J_{\alpha}$ is a (nonunique) direct sum of $n_{\alpha}$ minimal right invariant subspaces and that the family of all right invariant subspaces of $J_{\alpha}$ is order isomorphic to the family of all subspaces of an $n_{\alpha}$-dimensional vector space.

The finite-dimensional right invariant subspaces of $\mathcal{L}^{2}(G)$ furnish examples of what are called representations of $G$, that is, continuous homomorphisms of $G$ into the group of unitary transformations of a finite-dimensional Hilbert space into itself. Indeed if $M$ is such a subspace, $f \in M, a \in G$, and $U_{a}(f)=f_{a}$ where $f_{a}(x)=f(x a)$, then $U_{a}$ is unitary and the map $a \rightarrow U_{a}$ is a representation of $G$. The close and important connection between the theory of the representations of $G$ and the foregoing may be described briefly as follows. If $M^{(1)}$ and $M^{(2)}$ are minimal right invariant subspaces of the same $J_{\alpha}$, then the associated unitary representations $U^{(1)}$ and $U^{(2)}$ are unitary equivalent in the sense that there exists a unitary map $V$ of $M^{(1)}$ on $M^{(2)}$ such that $V U_{a}^{(1)} V^{-1}=U_{a}^{(2)}$ for all $a \in G$. Moreover any unitary representation $a \rightarrow U_{a}$ of $G$ which is irreducible in the sense that there is no subspace invariant under all $U_{a}$ is unitary equivalent to the representations associated with one and only of the $J_{\alpha}$. If $\phi_{1}, \phi_{2}, \cdots$ is a basis in the space in which the $U_{a}$ act, then the "matrix elements" $\left(U_{a} \phi_{i}, \phi_{j}\right)$ are continuous complex-valued functions defined on $G$. Their linear span is independent of the basis and in fact coincides with $\Im_{\alpha}$. It follows that the matrix elements of the irreducible representations of $G$ are dense in $\mathcal{L}^{2}(G)$, and it is in this form that the Peter-Weyl theorem is often quoted.

Since $\mathcal{L}^{2}(G)$ is the direct sum of the $\mathfrak{J}_{\alpha}$, each $f \in \mathcal{L}^{2}(G)$ is of the form $f=\sum_{\alpha} f_{\alpha}$ where $f_{\alpha} \in J_{\alpha}$. If we use representations, a compact and suggestive formula for $f_{\alpha}$ may be obtained. If $U_{f}^{\alpha}$ is defined for each $\alpha$ and each $f \in \mathcal{L}^{2}(G)$ by the formula

$$
U_{f}^{\alpha}=\int U_{x^{-1}}^{\alpha} f(x) d x
$$

where $U^{\alpha}$ is associated with $J_{\alpha}$, then

$$
f_{\alpha}=n_{\alpha} \text { Trace }\left[\begin{array}{ll}
U_{x}^{\alpha} & U_{f}^{\alpha}
\end{array}\right] \text {. }
$$

This last equality implies and is implied by the equality

$$
\int|f(x)|^{2} d x=\sum_{\alpha} n_{\alpha} \text { Trace }\left[U_{f}^{\alpha}\left(U_{f}^{\alpha}\right)^{*}\right],
$$

which may be regarded as a generalization of Parseval's equation in the theory of Fourier series. 
5. Almost periodic functions. Bochner's reformulation [4] of Bohr's definition of almost periodic functions on the line makes sense for arbitrary groups. For compact groups all the continuous functions turn out to be almost periodic. J. von Neumann in a celebrated paper [54] showed that the Peter-Weyl theory for continuous functions on a compact group could be obtained as a special case of an analogous theory of continuous almost periodic functions on an arbitrary topological group. ${ }^{8}$ When the group is the real line this theory reduces to that of Bohr. A central tool is a mean value defined for each almost periodic function and taking the place of the Haar integral. The main result asserts that the almost periodic functions on a group are related to the minimal finite-dimensional invariant subspaces of continuous functions and hence to the representations of the group just as are the continuous functions on a compact group. Later A. Weil ${ }^{9}[76 ; 78]$ showed that the theory of von Neumann could be deduced from the Peter-Weyl theory. More precisely he showed that for an arbitrary topological group $G$ there exists a continuous homomorphism $\phi$ of $G$ onto a dense subgroup of a certain compact group $K$ with the property that a function $f$ on $G$ is almost periodic if and only if there exists a continuous $g$ on $K$ such that $f(x)=g(\phi(x))$.

\section{Abelian Groups}

6. Duality and the Plancherel theorem. The theory of compact groups assumes an especially simple form when the group $G$ is Abelian as well as compact. The irreducible representations are all one-dimensional and so are simply continuous homomorphisms of $G$ into the group of complex numbers of modulus one, that is, characters of $G$. The minimal invariant subspace $J_{\alpha}$ of $\mathcal{L}^{2}(G)$ associated with the representation defined by the character $y_{\alpha}$ is simply the onedimensional subspace of all complex multiples of $y_{\alpha}$. Thus the expansion formula becomes $f(x)=\sum c_{y} y(x)$ where $c_{y}=\int f(x) \overline{y(x)} d x$ and the summation is over all characters $y$. Convergence is of course in the sense of the $\mathcal{L}^{2}(G)$ norm, that is, "in the mean."

It was apparently first noticed by A. Weil [78] that these formulae could be given a much more symmetric form and generalized to arbitrary locally compact Abelian groups by making use of the Pontrjagin-van Kampen duality theory $[62 ; 63 ; 39]$ for such groups. If $G$ is locally compact and Abelian, then the set $G^{\wedge}$ of all characters on $G$ is itself an Abelian group under ordinary pointwise multiplication.

${ }^{8}$ Cf. also Weyl [79].

${ }^{9}$ Cf. also Freudenthal [9], van Kampen [40], Pontrjagin [61] and Tannaka [74]. 
Moreover it is locally compact in a certain natural topology. Now if $y \in G^{\wedge}$ and $x \in G$, then for fixed $x, y(x)$ looked at as a function of $y$ is a character of $G^{-}$. The principal theorem of the Pontrjagin-van Kampen theory asserts that every character of $G^{-}$is uniquely obtainable in this way from an element of $G$ and that the topology of $G$ regarded as $G^{\wedge}$ is the same as the given topology of $G$. Thus there is a natural pairing of locally compact Abelian groups such that each member of a pair is the character group of the other. Because of the symmetry of the relationship between $G$ and $G^{\wedge}$ it is customary to write $(x, y)$ instead of $y(x)$. The resulting function of two variables is sometimes called the character function. If $G$ is the additive group of the real line, $G^{-}$is also isomorphic to the additive group of the real line and the character function may be taken to be such that $(x, y)=\exp (i x y)$. If $G$ is the additive group of the reals modulo $2 \pi$, then $G$ is isomorphic to the additive group of the integers and $(x, y)=\exp (i n x)$ where $n$ is an integer. In general the character group of a compact group is discrete and that of a discrete group compact.

Let us return to the formulae $f(x)=\sum_{y} c_{y} y(x)$ and $c_{y}=\int f(x) \overline{y(x)} d x$. Setting $c_{y}=f^{\wedge}(y)$ to emphasize the fact that the set of coefficients is a function on $G^{\Upsilon}$, we may rewrite these formulae in the form

$$
f(x)=\int f^{\wedge}(y)(x, y) d y \text { and } \quad f^{\frown}(y)=\int f(x) \overline{(x, y)} d x
$$

where the integrals are taken with respect to the Haar measures in $G$ and $G^{\wedge}$. Indeed, since $G^{\wedge}$ is discrete, the Haar measure of a point may be taken to be one and summation over the group elements is the same as integrating over the group. In this form, however, the formulae are meaningful for arbitrary locally compact groups and A. Weil in [78] has established their validity for suitably restricted functions $f$. In particular the $f^{\wedge}$ for $f \in \mathcal{L}^{1}(G) \cap \mathcal{L}^{2}(G)$ are dense in $\mathcal{L}^{2}\left(G^{\wedge}\right)$ and (for suitable choice of the Haar measure in $G$ ) $\int|f(x)|^{2} d x=\int\left|f^{\wedge}(y)\right|^{2} d y$ for all such $f$. Thus the map $f \rightarrow f^{\wedge}$ may be uniquely extended by continuity to give a natural norm preserving linear map of all of $\mathcal{L}^{2}(G)$ onto all of $\mathcal{L}^{2}\left(G^{\wedge}\right)$. This "Plancherel theorem" for locally compact Abelian groups reduces to the classical Plancherel theorem for Fourier transforms when $G$ is taken to be the additive group of the real line and to the Parseval relation for Fourier series plus the Riesz-Fisher theorem when $G$ is taken to be the real line modulo $2 \pi$. For arbitrary locally compact Abelian groups the generalized Fourier transform $f \rightarrow f^{\wedge}$ makes possible a quite satisfactory solution of the invariant subspaces problem for $\mathcal{L}^{2}(G)$. In 
fact it is not hard to show that the Fourier transform of a translation invariant closed subspace of $\mathcal{L}^{2}(G)$ is the set of all members of $\mathcal{L}^{2}\left(G^{\wedge}\right)$ which vanish (almost everywhere) outside of some measurable subset of $G^{\wedge}$ and conversely. Thus there is a one-to-one inclusion preserving correspondence between the closed invariant subspaces of $\mathcal{L}^{2}(G)$ and the measurable subsets mod null sets of $G^{\text {}}$.

7. Normed rings and $\mathscr{L}^{1}(G)$. The generalized Fourier transform also arises naturally when Gelfand's theory of normed rings (Banach algebras) is applied to the algebra $A_{G}$ obtained by adjoining a unit to the algebra $\mathcal{L}^{1}(G)$. For an arbitrary Banach algebra $\mathcal{A}$ the Gelfand theory gives a canonical homomorphism $f \rightarrow f^{0}$ where $f \in \mathcal{C} A$ and $f^{0}=f^{0}(M)$ is a member of the ring of continuous complex-valued functions defined on the compact space $\mathcal{X}$ of all maximal ideals in $\mathcal{A}$. When $\mathcal{A}=\mathcal{A}_{G}$, the maximal ideals other than $\mathcal{L}^{1}(G)$ itself are in oneto-one correspondence with the characters of $G$ in such a manner that for each $f \in \mathcal{L}^{1}(G)$ and each $M \in \mathcal{X}-\left\{\mathcal{L}^{1}(G)\right\}, f^{0}(M)=\int f(x) y_{M}(x) d x$ where $y_{M}$ is the character corresponding to $M$. Thus functions on $\mathscr{X}$ may be identified with functions on $G^{\widehat{ }}$ and the canonical mapping $f \rightarrow f^{0}$ reduces to the Fourier transform. The fact that $f^{0}=0$ implies that $f^{0}=0$ is equivalent to the fact that $A_{G}$ is semisimple. The Fourier transform from this point of view seems to have been introduced independently by Gelfand and Raikov [20] on the one hand and I. E. Segal [68] on the other without knowledge of Weil's work. The application of the normed ring point of view to functions in $\mathcal{L}^{2}(G)$ and the proof of the Plancherel theorem seems to have been first carried out by M. Krein [43].

The invariant subspaces problem for $\mathcal{L}^{1}(G)$ is much more difficult than that for $\mathcal{L}^{2}(G)$ and so far is only very incompletely solved even when $G$ is the real line. It is natural in view of the $\mathcal{L}^{2}$ theory to attempt to set up a correspondence between the closed ideals in $\mathcal{L}^{1}(G)$ and subsets of $G^{\Upsilon}$. Following Segal, ${ }^{10}$ who seems to have been the first to attack the problem for general groups $[68 ; 69]$, let us define the hull of a closed ideal $\mathfrak{J}$ in $\mathcal{L}^{1}(G)$ as the set $h(\mathfrak{I})$ of all characters which define maximal ideals containing $J$. Let us define the kernel of a subset $A$ of $G^{\wedge}$ as the intersection $k(A)$ of the maximal ideals defined by members of $A$. Then $k(h(J))$ the kernel of the hull of $J$ contains $J$. If it were true that $J=k(h(J))$ for all $J$, then there would be a one-to-one inclusion inverting correspondence between the closed invariant subspaces of $\mathcal{L}^{1}(G)$ and the closed subsets of $G$ and a

${ }^{10}$ Results similar to those of Segal have been obtained independently by Godement [26]. 
satisfactory solution of the invariant subspaces problem would be at hand. Unfortunately L. Schwartz [67] has recently shown that this equality need not hold; specifically whenever $G$ is Euclidean $n$ space with $n \geqq 3$. I. E. Segal has shown on the other hand that it is always true that $J$ is "approximately" equal to $k(h(J))$. More precisely if $O$ is any open set containing $h(J)$, then $k(O) \subseteq J$. It follows that whenever $h(J)$ is empty, then $J=k(h(J))$. This result for the real line implies Wiener's general Tauberian theorem and his theorem about spans of functions in $\mathcal{L}^{1}(G)$. In fact the nontrivial half of one form of the general Tauberian theorem (Theorem 4 of [80]) may be stated as follows. Let $f$ be a bounded measurable function on the line and let $c$ be a complex number. Let $J$ be the class of all functions $K$ in $\mathcal{L}^{1}(-\infty, \infty)$ such that $\lim _{x \rightarrow \infty} \int_{-\infty}^{\infty} K(x-y) f(y) d y=c \int_{-\infty}^{\infty} K(x) d x$. Then if $\exists$ contains a function $K_{1}$ whose Fourier transform never vanishes, $J$ must coincide with $\mathcal{L}^{1}(-\infty, \infty)$. Now it is routine to verify that $J$ is a closed ideal. Moreover the fact that the Fourier transform of $K_{1}$ never vanishes is equivalent to the fact that $K_{1}$ is not contained in the maximal ideal defined by any character exp $(i x y)$. This implies that the hull of $J$ is empty and hence that $k(h(J))=\mathcal{L}^{1}(-\infty, \infty)$. In Segal's paper, which also contains the developments necessary to obtain the other form of Wiener's theorem, this theorem is generalized in another direction: when $G$ is the real line, then $J=k(h(J))$ whenever $h(J)$ is the union of an open set and a closed set without limit points. I. Kaplansky [41] has recently extended this last result to a large class of locally compact Abelian groups and shown that for arbitrary locally compact Abelian groups $J=k(h(J))$ whenever $h(J)$ has only a finite number of elements.

8. The Laplace transform and connections with the theory of functions of a complex variable. Further connections of the theory of locally compact Abelian groups with classical analysis appear when other invariant classes of functions on a group are considered. Specifically it is possible in a great variety of ways to choose a measure $\mu$ on $G$ which is different from Haar measure but absolutely continuous with respect to it in such a manner that $\mathcal{L}^{1}(\mu, G)$ is closed with respect to convolution. ${ }^{11}$ The maximal ideals in the resulting Banach algebra are no longer in one-to-one correspondence with the characters of $G$ but with what may be called generalized characters, that is, continuous homomorphisms of $G$ into the multiplicative group of all nonzero complex numbers. Moreover, for particular such Banach algebras not all generalized characters need appear. Every

\footnotetext{
${ }^{11}$ Convolution being defined as usual using Haar measure and not $\mu$.
} 
generalized character is uniquely a product of an ordinary character and a function of the form exp $(l)$ where $l$ is real-valued and has the property that $l(x y)=l(x)+l(y)$ for all $x$ and $y$ in $G$, that is, is a linear functional. If we let $\bar{G}$ denote the vector space of all continuous linear functionals on $G$, the maximal ideals of $\mathcal{L}^{1}(\mu, G)$ will be in one-to-one correspondence with the members of a subset of $G^{\wedge} \times \bar{G}$. It can be shown that this subset is always of the form $G^{\wedge} \times K$ where $K$ is a suitable convex subset of the vector space $\bar{G}$. The canonical mapping taking each $f \in \mathcal{L}^{1}(\mu, G)$ into a function on the maximal ideals is then a mapping of functions on $G$ into functions on $G^{\wedge} \times K$. If $f^{0}$ is the map of $f$ and $y$ and $l$ are members of $G$ and $k$ respectively, then

$$
f^{0}(y, l)=\int f(x)(x, y) \exp (l(x)) d x .
$$

If $G$ is the group of integers, then $G^{-}$is the group of complex numbers of unit modulus and $\bar{G}$ is a one-dimensional vector space. A convex subset of $\bar{G}$ is an interval so that the functions $f^{0}$ are defined on the direct product of a circle with an interval, that is, an annulus. It is easy to see that the mapping from sequences to functions defined in an annulus thus obtained coincides with the corresponding mapping defined by the Laurent series. If $G$ is the real line, then $G^{\wedge} \times K$ is a strip and $f \rightarrow f^{0}$ is the two-sided Laplace transform. In the general case it is possible to introduce a notion of directional differentiation for functions defined on $G^{\wedge} \times K$ and a relationship between directions of differentiation which leads to a notion of analyticity for functions on $G^{\wedge} \times K$. The generalized Laplace transforms, that is, the functions $f^{0}$ defined by $\left({ }^{*}\right)$, then turn out to be analytic functions on $G^{\wedge} \times K$. In this way at least some of the main notions in the theory of functions of a complex variable arise as special cases of more general notions occurring naturally in the theory of locally compact Abelian groups. One is led to conjecture that there will be general theorems about such groups which will unify and illuminate appropriate portions of the classical theory of functions of one or more complex variables. These matters have been discussed in outline by the author in [46]. A preliminary example of what can be done is the following (unpublished) generalization of Hadamard's three circles theorem. If $f$ is analytic in $G^{\wedge} \times K$ and is bounded in $y$ for each fixed $l$ in $K$, then $\log \left(\sup _{y} \in \sigma^{-}|f(y, l)|\right)$ is a convex function of $l$ on the convex subset $K$ of the vector space $\bar{G}$. This theorem includes Hadamard's three circles theorem for several complex variables and hence Thorin's [75] generalization of the M. Riesz convexity theorem. 
The ideal theory of the rings $\mathcal{L}^{1}(\mu, G)$ seems not to have been investigated at all in the general case, although Gelfand in [11] has defined such rings for the real line and integer groups and these rings have been further discussed in [22]. The fact that the functions $f^{0}$ are analytic instead of merely continuous can naturally be expected to produce many divergences from the theory of $\mathcal{L}^{1}(G)$. In particular the theorem of Kaplansky to the effect that an ideal whose hull is only a single point must be maximal certainly cannot be expected to carry over. In fact for each point $y, l$ "interior" to $G$ ^ $\times K$ and each "direction of differentiation" $v$ the set of all $f \in \mathcal{L}^{1}(G, \mu)$ for which $f_{v}(y, l)$ $=f(y, l)=0$ will be an ideal which will not be maximal but will be contained in only one maximal ideal. A number of questions suggest themselves. Is every ideal with a finite hull maximal provided that every point of the hull is an extreme point of the boundary of $G \frown \times K$; that is, a point of the form $y, l$ where $l$ is an extreme point of $K$ ? Is every counter example to the proposed analogue of Kaplansky's theorem of the form just described (with higher order derivatives of course)? Do the extreme points of the boundary of $G^{-} \times K$ constitute the boundary in the sense of Silov [22] of the set of maximal ideals? These are questions which the author intends to investigate and discuss elsewhere. The last has been answered in the affirmative in special cases by Silov.

The work of Laurent Schwartz [66] on mean periodic functions has close connections with the foregoing. Schwartz considers the set of all continuous functions on the real line topologized so that convergence is uniform convergence on compact sets. The dual of this topological vector space is the set of all complex-valued Borel measures on the line which are concentrated in compact sets. These measures form a ring under convolution and there is a one-to-one inclusion inverting correspondence between the closed translation invariant subspaces of continuous functions and the closed ideals in this ring. This ring is of the same general nature as those considered above and its closed maximal ideals are in one-to-one correspondence with the points of the complex plane. The canonical homomorphism carries this ring into a multiplicative ring of entire functions. Schwartz shows essentially that every closed primary ideal in the original ring is determined by a complex number $a$ and a non-negative integer $k$ and consists of all members such that the corresponding entire function and its first $k$ derivatives vanish at the point $a$. He shows furthermore that every closed ideal is an intersection of primary ones, thus determining all possible closed ideals in a very explicit fashion. 


\section{General Locally Compact Groups}

9. Preliminaries. The theory of locally compact groups which are neither compact nor Abelian is much less well developed than the theories of either of these special classes. Even the $\mathcal{L}^{2}$ theory, which is more or less complete for compact and Abelian groups, is in a rather unsatisfactory state for more general groups. A part of the trouble may be ascribed to the fact that when the group is neither compact nor Abelian one must deal with "continuous" decompositions into "multi-dimensional parts." Thus neither the discreteness of the compact case nor the one-dimensionality of the Abelian case is available and there is a compounding of difficulties. A perhaps more serious source of difficulty is the fact that it is no longer possible to confine attention to finite-dimensional representations. There are locally compact groups - the group of all two-by-two complex matrices with determinant one is an example-which admit no nontrivial finite-dimensional unitary representations at all. It is thus necessary to turn to representations by unitary operators in infinitedimensional Hilbert spaces and to analyze the more complicated phenomena which almost always appear when one makes a transition from the finite to the infinite.

10. Positive definite functions and the existence of sufficiently many irreducible representations. Let $\mathcal{L}^{2}(G)$ denote the Hilbert space of all complex-valued functions on $G$ which are square summable with respect to right invariant Haar measure in $G$. For each $f \in \mathcal{L}^{2}(G)$ and each $s \in G$ let $U_{s}(f)$ be the function $g \in \mathcal{L}^{2}(G)$ such that $g(x)=f(x s)$ for all $x \in G$. Then $f \rightarrow U_{s}(f)$ is a unitary operator. Moreover the map $s \rightarrow U_{s}$ is a continuous representation of $G$ in the sense ${ }^{12}$ that it is a homomorphism of $G$ into the group of all unitary operators in $\mathcal{L}^{2}(G)$ such that $\left(U_{s}(f), g\right)$ is continuous in $s$ for all $f$ and $g$ in $\mathcal{L}^{2}(G)$. This representation is called the right regular representation of $G$. Its existence makes it clear that every locally compact group has sufficiently many representations to distinguish between any two group elements if infinite-dimensional representations are allowed. It does not follow at once that there need exist sufficiently many (or even any) nontrivial irreducible representations. This much less trivial existence theorem was first proved by Gelfand and Raikov [21]. Here irreducibility must be taken to mean that no closed invariant sub-

${ }^{12}$ Actually $\left(U_{s}(f), g\right)$ is continuous for all $f$ and $g$ if and only if $U_{s}(f)$ is continuous for all $f$. In the sequel we shall use the terms representation and unitary representation interchangeably to denote a unitary representation which has one and hence both of these equivalent properties. 
spaces exist. There are many examples showing that the theorem is false if irreducibility is taken to mean that no invariant subspaces at all exist.

In order to prove this theorem Gelfand and Raikov exploit a close relationship which exists between representations and those continuous functions on $G$ which have the property of "positive definiteness." Let $U\left(x \rightarrow U_{x}\right)$ be any representation of $G$. Let $\phi$ be any element in the associated Hilbert space and let $f$ be the continuous complex-valued function defined on $G$ by the equation $f(x)$ $=\left(U_{x}(\phi), \phi\right)$. It is trivial to verify that $f$ has the following property. If $x_{1}, x_{2}, \cdots, x_{n}$ is any finite subset of $G$ and $c_{1}, c_{2}, \cdots, c_{n}$ is a set of complex numbers, then $\sum_{i, j=1}^{n} c_{i} \bar{c}_{j} f\left(x_{i} x_{j}^{-1}\right) \geqq 0$. Any function with this property is said to be positive definite. It is not difficult to show that conversely every continuous positive definite function is obtainable in this manner from a suitable representation of $G$. This representation may always be chosen so as to be cyclic in the sense that, for some $\phi$, the $U_{x}(\phi)$ span a dense subspace of the associated Hilbert space. Moreover, if this is done, the correspondence is one-to-one in the following sense: $\left(U_{x}(\phi), \phi\right)$ and $\left(U_{x}^{\prime}\left(\phi^{\prime}\right), \phi^{\prime}\right)$ are the same for all $x$ in $G$ if and only if $U$ and $U^{\prime}$ are unitary equivalent via a unitary transformation which carries $\phi$ into $\phi^{\prime}$. The representation associated with the continuous positive definite function $f$ is irreducible if and only if $f$ is "elementary" in the sense that it is not of the form $f_{1}+f_{2}$ where $f_{1}$ and $f_{2}$ are linearly independent continuous positive definite functions. The existence of sufficiently many elementary positive definite functions on $G$ and hence of sufficiently many irreducible representations is made to depend upon a theorem of Krein and Milman [44] asserting the existence of sufficiently many extreme points in certain convex sets. The convex set to which this theorem is applied is the set of all continuous positive definite functions $f$ for which $f(e)=1$. (Here $e$ is the identity of $G$.)

Functions on $G$ of the form $\left(U_{x}(\phi), \psi\right)$, where $U\left(x \rightarrow U_{x}\right)$ is a representation of $G$ and $\phi$ and $\psi$ are in the Hilbert space in which the $U_{x}$ act, are finite linear combinations with complex coefficients of functions of the form $\left(U_{x}(\phi), \phi\right)$. Thus if $G$ is compact, the finite sums of elements from the ideals $J_{\alpha}$ are exactly the finite linear combinations of elementary positive definite functions. R. Godement in [27] has generalized one form of the Peter-Weyl theorem to arbitrary locally compact groups by applying the theory of Gelfand and Raikov to show that every continuous function on such a group can be uniformly approximated on every compact set by finite linear combinations of elementary positive definite functions. Actually Godement 
developed much of the theory of positive definite functions independently of Gelfand and Raikov. His paper [27] is a complete exposition of the work of Gelfand, Raikov, and himself, and contains many theorems about the behavior of positive definite functions on groups in addition to those described here.

11. The reducibility of representations. Let $U$ be a finite-dimensional representation of a group $G$ and let $H$ be the underlying (finite-dimensional) Hilbert space. It is well known and easily proved that $H$ is a direct sum of orthogonal subspaces in each of which $U$ induces an irreducible representation. This decomposition is unique in the sense that the irreducible summands in any two such are unitary equivalent in some order. Actually the only nonuniqueness lies in the decomposition of direct sums of mutually unitary equivalent irreducible representations. In general the direct sum of all subspaces of $H$ in which the "same" irreducible representation is induced is independent of the original decomposition. For infinitedimensional unitary representations a similar theorem holds provided that $G$ is compact $[6 ; 37]$. When $G$ is not necessarily compact it is not difficult to see that no such decomposition need exist. The regular representation of a noncompact locally compact Abelian group is an example. Any nonzero closed invariant subspace of $\mathcal{L}^{2}(G)$ is readily seen to have proper closed invariant subspaces. On the other hand, the Plancherel theorem shows that $\mathcal{L}^{2}(G)$ may be mapped unitarily onto $\mathcal{L}^{2}\left(G^{-}\right)$in such a manner that translation by $s$ goes over into multiplication by $(s, y)$ where $y$ is a generic element of $G$. Roughly speaking the regular representation of $G$ appears to be a sort of "continuous direct sum" of the one-dimensional (and hence irreducible) representations defined by the various characters.

Clearly what is needed is a generalization of the notion of direct sum which will include as a special case a precise notion of "continuous direct sum" and which can be used to decompose arbitrary representations into irreducible parts. A theory of such a notion has been developed by J. von Neumann [58] in order to decompose rings of operators into "factors" and the von Neumann theory has been applied to the theory of representations of separable locally compact groups of F. I. Mautner $[48 ; 49]$. Slightly reformulated, ${ }^{13}$ the basic notion may be described as follows. Let $S$ be a set and let $\mathcal{F}$ be a countably generated $\sigma$ field of subsets of $S$. Let $\mu$ be a countably additive measure defined on $\mathcal{F}$. For each $n=1,2, \cdots, \infty$, let $\mathfrak{F C}_{n}$ be an $n$-dimensional separable Hilbert space. Let $d$ be a measurable

${ }^{13}$ Cf. Godement [29] for an alternative approach. 
function from $S$ to the positive integers and $\infty$. Let $\mathcal{H}(S, \mu, d)$ be the set of all functions from $S$ to $\mathcal{F}_{\infty} \cup \mathcal{H}_{1} \cup \mathcal{F C}_{2} \cup \ldots$ such that: (a) $f(x) \in \mathcal{F}_{d(x)}$ for all $x$, (b) for each $n=1,2, \cdots, \infty$ and each $v \in \mathfrak{H}_{n}$, $(f(x), v)$ is a measurable function of $x$ for $x$ in $d^{-1}(n)$, (c) $\int(f(x), f(x)) d \mu(x)<\infty . \mathfrak{C}(S, \mu, d)$ becomes a Hilbert space when the inner product of two members $f$ and $g$ is defined by the equation $[f, g]=\int(f(x), g(x)) d \mu(x)$. Now suppose that a representation $U^{(x)}$ $\left(s \rightarrow U_{s}^{(x)}\right)$ of a group $G$ in $\mathfrak{T C}_{d(x)}$ is given for each $x \in S$. If $U^{(x)}$ depends measurably on $x$ in the sense that, for each $n,\left(U_{s}^{(x)}\left(v_{1}\right), v_{2}\right)$ is measurable as a numerical function on $d^{-1}(n)$ for each $v_{1}$ and $v_{2}$ in $\mathcal{K}_{n}$, then there is a unique representation $U$ of $G$ in $\mathfrak{H C}(S, \mu, d)$ such that for all $f$ in $\mathcal{H}(S, \mu, d)$, the equation $\left(U_{s} f\right)(x)=U_{s}^{(x)}(f(x))$ holds for each $s$ and almost all $x$. $U$ (or any representation unitary equivalent to $U$ ) is said to be the generalized direct sum of the representations $U^{(x)}$ with respect to the measure $\mu$. It is clear that this notion reduces to that of ordinary direct sum whenever $S$ is a discrete measure space.

This notion is adequate for the purpose indicated above. One of the principal results of [48] asserts ${ }^{14}$ that an arbitrary representation (in a separable Hilbert space) of a separable locally compact group $G$ is a generalized direct sum of irreducible representations of $G$. Unfortunately this result does not reduce the study of arbitrary representations to that of irreducible representations, for the decomposition in question can be rather badly nonunique. On the other hand, there is a generally possible unique reduction into components which, while not irreducible, nevertheless give evidence of being the proper fundamental building blocks. The details are as follows. Let $U$ be a generalized direct sum of representations $U^{(x)}$ with the associated measure space $S$. For each measurable subset $E$ of $S$, let $P_{E}$ be the projection which takes $f \in \mathcal{F}(S, \mu, d)$ into $\phi_{E} f$ where $\phi_{E}$ is the characteristic function of $E$. Then the set $\mathcal{E}$ of all $P_{E}$ is a $\sigma$-Boolean algebra of projections in the sense that the identity $I$ is in $\mathcal{E}$, that whenever $P_{1}, P_{2}, \cdots$ are in $\varepsilon$ then $P_{1} P_{2}=P_{2} P_{1} \in \mathcal{E}$ and $I-P_{1} \in \mathcal{E}$, and that if $P_{i} P_{j}=0$ then $P_{1} \dot{+} P_{2} \dot{+} \cdots \in \mathcal{E}$. Moreover $\varepsilon$ commutes with $U$ in the sense that $P U_{s}=U_{s} P$ for all $P \in \mathcal{E}$ and all $s \in G$. Conversely, given any $\sigma$-Boolean algebra $\varepsilon$ which commutes with a representation $U$, it is possible to decompose $U$ as a generalized direct sum in such a manner that $\varepsilon$ is the $\sigma$-Boolean algebra defined by the decomposition. Moreover, modulo null sets and replacement of $\mu$ by an equivalent measure, the decomposition is uniquely determined. We may speak of the decomposition defined by $\mathcal{E}$. This decomposi-

${ }^{14}$ Note that this furnishes an alternative proof of the existence of sufficiently many irreducible representations when $G$ is separable. 
tion will have almost all of its parts irreducible if and only if $\varepsilon$ is maximal; that is, if and only if whenever $\varepsilon^{\prime} \supseteq \mathcal{E}$ where $\varepsilon^{\prime}$ is also a $\sigma$-Boolean algebra commuting with $U$, then $\varepsilon=\varepsilon^{\prime}$. In general there will be a number of distinct maximal $\mathcal{E}$ 's commuting with a given $U$. This is so even for finite-dimensional representations, and occurs there when and only when some irreducible components are repeated. Consider now the $\sigma$-Boolean algebra $\varepsilon_{0}$ defined as follows. $E \in \mathcal{E}_{0}$ if and only if $E$ commutes with all $U_{s}$ and with all operators $A$ which commute with all $U_{s} . \mathcal{E}_{0}$ is contained in every maximal $\mathcal{E}$ and is uniquely determined by $U$. The decomposition defined by $\varepsilon_{0}$ has the property that almost every component has as its $\varepsilon_{0}$ the set consisting of 0 and the identity. We shall call a representation $V$ whose $\varepsilon_{0}$ is trivial in this sense a factor ${ }^{15}$ representation. Thus every representation has an essentially unique decomposition as a generalized direct sum of factor representations.

In the finite-dimensional case a representation is a factor representation if and only if it is a direct sum of replicas of the same irreducible representation. In the infinite-dimensional case things are considerably more complicated. Given a representation $U$, let $\left\{U_{s}\right\}^{\prime}$ denote the set of all bounded operators $A$ in the underlying Hilbert space such that $A U_{s}=U_{s} A$ for all $s \in G$ and let $\left\{U_{s}\right\}^{\prime \prime}$ be similarly defined with $\left\{U_{s}\right\}$ replaced by $\left\{U_{s}\right\}^{\prime}$. Then $\left\{U_{s}\right\}^{\prime \prime}$ is an operator ring in the sense of Murray and von Neumann [52] and a necessary and sufficient condition that $U$ be a factor representation is that $\left\{U_{s}\right\}^{\prime \prime}$ be a factor, that is, that it contain only multiples of the identity in its center. Now Murray and von Neumann have studied factors in great detail and have classified them according to the behavior of a certain dimension function uniquely (up to a multiplicative constant) defined for the projections in any factor. The factor is said to be of type $\mathrm{I}_{n}, \mathrm{I}_{\infty}, \mathrm{II}_{1}, \mathrm{II}_{\infty}$, or $\mathrm{III}_{\infty}$ according as the (suitably normalized) dimension function takes on the values $1,2, \cdots, n$; $1,2, \cdots, \infty$, all real numbers between 0 and 1 , all non-negative real numbers and $\infty$, or just 0 and $\infty$. Factors of all types exist. It is shown in [49] that a factor representation is a generalized direct sum of replicas of the same irreducible representation if and only if the corresponding factor is of type $\mathrm{I}_{n}$ or $\mathrm{I}_{\infty}$. Moreover, this irreducible representation is uniquely determined. For factor representations not of type $I_{n}$ or $I_{\infty}$, however, the behavior can be very different indeed. Mautner [50] has given an example of a group whose regular representation is a factor representation of type $\mathrm{II}_{1}$ and has

${ }^{15}$ Because of the close connection with the Murray-von Neumann notion of factor for rings of operators. See below. Cf. Wigner [81]. 
found a decomposition of it into irreducible components in which uncountably many inequivalent representations occur. The author has shown (unpublished) that this representation has another decomposition into irreducible parts such that (a) no two representations in the decomposition are unitary equivalent and (b) no representation in the decomposition is unitary equivalent to any representation in Mautner's decomposition. On the other hand, general factor representations do share the following property of factor representations of types $I_{n}$ and $I_{\infty}$. A decomposition as a direct sum of two other representations is always such that one component is unitary equivalent to a discrete part of the other. To summarize: Every unitary representation of a separable locally compact group is a unique generalized direct sum of factor representations. Every factor representation of type $\mathrm{I}_{n}$ or $\mathrm{I}_{\infty}$ is a generalized direct sum (actually an ordinary discrete direct sum) of replicas of a uniquely determined irreducible representation. A factor representation not of type $I_{n}$ or $I_{\infty}$ can be decomposed into irreducible parts, but the decomposition may be wildly nonunique. The structure of such representations is very little understood.

There remains the question of the extent to which a representation is determined by the factor representations into which it may be decomposed. The first remark to be made is that it is not sufficient simply to list the representations which occur in the breakdownthe measure $\mu$ is itself of decisive importance. This is more or less clear from the fact that sets of representations of measure zero may be added and removed without altering the sum. A better appreciation of the situation may be obtained by considering what happens when $G$ is Abelian. Let $G \wedge$ be the character group of $G$. Let $\mu$ be any Borel meas-

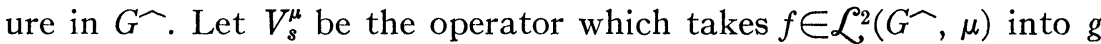
where $g(y)=(s, y) f(y)$ for all $y \in G$. Then $V^{\mu}\left(s \rightarrow V_{s}^{\mu}\right)$ is a unitary representation of $G$ and as a matter of fact every representation of $G$ in which there are "no multiplicities" is unitary equivalent to some $V^{\mu}$. Two such representations $V^{\mu}$ and $V^{\nu}$ are unitary equivalent if and only if $\mu$ and $\nu$ have the same null sets. When multiplicities are allowed, things are only slightly more complicated. Choose a Borel measure $\mu$ in $G^{\wedge}$ and a Borel function $d$ from $G^{\wedge}$ to the positive integers and $\infty$. Let $\mu_{n}(n=1,2, \cdots, \infty)$ be the unique Borel measure which agrees with $\mu$ on subsets of $d^{-1}(n)$ and is zero for subsets of the complement of $d^{-1}(n)$. Let $V^{\mu, d}$ be the direct sum of countably many replicas of $V^{\mu_{\infty}}$, one replica of $V^{\mu_{1}}$, two replicas of $V^{\mu_{2}}$, and so on. Then every representation of $G$ is unitary equivalent to some $V^{\mu, d}$, and $V^{\mu, d}$ and $V^{\nu, d^{\prime}}$ are unitary equivalent if and only if $\mu$ and $\nu$ 
have the same null sets and $d$ and $d^{\prime}$ are equal almost everywhere with respect to $\mu$ and $\nu$. The regular representation of $G$ has Haar measure for $\mu$ and $d(y) \equiv 1$. These facts about Abelian groups follow from the theory of multiplicity of spectra for normal operators in Hilbert space and the following spectral resolution theorem. If $s \rightarrow U_{s}$ is an arbitrary representation of the locally compact group $G$ by operators in a Hilbert space $\mathcal{H}$, then there exists a mapping $E \rightarrow P_{E}$ of the Borel subsets $E$ of $G$ into projections in $\mathcal{H}$ such that the countable Boolean operations are preserved and such that for all $\phi$ and $\psi$ in $\mathfrak{H C}\left(U_{s}(\phi), \psi\right)=\int(s, y) d\left[P_{y}(\phi), \psi\right]$ where integration is over $G$ with respect to the completely additive set function $E$ $\rightarrow\left(P_{E} \phi, \psi\right)$. This theorem was first proved by Stone [73] for the case in which $G$ is the additive group of the real line. It was extended to arbitrary locally compact Abelian groups independently by Ambrose, Godement, and Neumark $[1 ; 23 ; 59]$.

When $G$ is not necessarily Abelian and $U\left(s \rightarrow U_{s}\right)$ is a representation of $G$, we have seen that $U$ is an essentially unique generalized direct sum of factor representations $U^{(y)}$ with respect to a measure $\mu$. In view of the foregoing it is natural to conjecture that two representations $U$ and $V$ are unitary equivalent if and only if almost all the $U^{(y)}$ can be mapped one-to-one onto almost all the $V^{(z)}$ in such a way that null sets and measurability are preserved and so that the image of each $U^{(y)}$ is unitary equivalent to it. So far as the author knows no proof of this theorem is at present available. It seems likely, however, that the methods of von Neumann and Mautner will be adequate to produce one. It is to be observed that the "only if" part of this conjecture cannot be expected to be true unless the $U^{(y)}$ and $V^{(z)}$ are known to arise from a canonical decomposition into factor representations defined by an $\varepsilon_{0}$ as described above. This follows from the fact noted above that a generalized direct sum of distinct irreducible representations can be a factor representation. The question: What measure spaces of factor representations "add up" to factor representations remains to be investigated. Until something substantial about its answer is known the theory of general representations can hardly be regarded as reduced to that of factor representations even when the above conjecture has been proved.

For a fully satisfactory theory of the decomposability of representations, even more than an answer to the questions of the last paragraph would be desirable. One would want a sort of "dual object"-a topological space $G^{\sim}$, the points of which would be classes ${ }^{16}$

${ }^{16}$ Given two factor representations $U$ and $V$, it can be shown that either (a) no discrete direct summand of one is unitary equivalent to any discrete direct summand 
of factor representations. One would then hope for a theorem setting up a one-to-one correspondence between unitary equivalence classes of representations of $G$ on the one hand, and on the other systems consisting of a family of null sets in $G^{\sim}$ and a function assigning one member of its class to each member of $G^{\sim}$. The prognosis for such a theorem for general locally compact groups is not encouraging. The author has studied in some detail the representations of semi direct products of locally compact Abelian groups. ${ }^{17}$ For a large subclass of these groups a complete analysis of the representations can be given, and there is little doubt that for members of this subclass suitable dual objects can be found. However, for semi direct products not in this subclass, irreducible representations and a fortiori factor representations exist in a bewildering profusion which so far has defied exhaustive analysis. Certainly it is difficult to imagine their being all included in any sort of well behaved dual object. On the other hand, there are a number of groups other than the compact and Abelian ones for which dual objects and corresponding decomposition theorems are available. Gelfand and Neumark [15] have shown that this is the case for the group of all two-by-two complex matrices of determinant one. More generally, I. Kaplansky in an as yet unpublished work has obtained such a theorem for any "completely continuously representable" or "CCR" group; that is, for any group $G$ which has the following property: If $f \in \mathcal{L}^{1}(G)$ and $U$ is an irreducible representation of $G$, then $\int f(x) U_{x} d x$ is a completely continuous operator. ${ }^{18}$ It can be shown that such groups admit only factor representations of type $\mathrm{I}_{n}$ and $\mathrm{I}_{\infty}$. It is not inconceivable that, conversely, every group all of whose factor representations are of type $I$ is a CCR group. If so, Kaplansky's result may be regarded as solving the reducibility problem modulo the pathology of nontype I factors.

12. $\mathcal{L}^{2}(G)$ and the Plancherel theorem for unimodular groups. In this section we shall deal throughout with locally compact groups which are not only separable but also are "unimodular" in the sense

of the other or (b) given any discrete direct summand of $U$ and any discrete direct summand of $V$ one is unitary equivalent to a discrete direct summand of the other. When (b) holds we may say that $U$ and $V$ are quasi unitary equivalent. The relation of quasi unitary equivalence is an equivalence relation in the technical sense and divides factor representations into classes of mutually quasi unitary equivalent elements.

${ }^{17}$ If $G$ admits a closed normal subgroup $G_{1}$ and a second closed subgroup $G_{2}$ such that $G_{1} \cap G_{2}=e$ and $G_{1} G_{2}=G$, then $G$ is said to be a semi direct product of $G_{1}$ and $G_{2}$.

${ }^{18} \mathrm{It}$ is irrelevant whether left or right invariant Haar measure is used here. 
that left and right Haar measures coincide. For such groups there is a single $\mathcal{L}^{2}(G)$ which is the underlying space for both the right and left regular representations. The family $\mathcal{F}$ of closed subspaces invariant under both right and left translation coincides with the family of closed subspaces which simultaneously reduce the right and left regular representations. A recent result ${ }^{19}$ of Segal [71] analyzes the internal structure of this family in quite a satisfactory manner. This result (proved earlier by Murray and von Neumann [53] for discrete groups) asserts that the weakly closed self-adjoint operator rings $R_{l}$ and $R_{r}$ generated by the unitary operators in the left and right regular representations respectively are commutators of one another; that is, $A \in R_{l}$ if and only if it commutes with all members of $R_{r}$ and vice versa. It implies in particular that the projections on the members of $\mathcal{F}$ are precisely the projections in the common center of $R_{r}$ and $R_{l}$. Thus the projections on the members of $\mathcal{F}$ not only commute with one another, but form a $\sigma$-Boolean algebra of projections. More than this is true. The Segal result also implies that this Boolean algebra is identical with the one which defines the decomposition of the right (left) regular representation into factor components. Let $S$ be a measure space of factor representations effecting this decomposition. It follows that the closed two-sided invariant subspaces of $\mathcal{L}^{2}(G)$ are in one-to-one inclusion preserving correspondence with the measurable sets mod null sets of $S$. Thus a theorem on the structure of the family of two-sided invariant closed subspaces of $\mathcal{L}^{2}(G)$ is available which is quite analogous to that which has already been discussed for compact and Abelian groups.

A more difficult problem is that of discussing the decomposition of individual functions in $\mathcal{L}^{2}(G)$; that is, proving a generalization of the Plancherel theorem. For compact groups, it will be recalled, the basic fact is that $\int|f(x)|^{2} d x=\sum_{\alpha} d_{\alpha}$ Trace $\left(U_{f}^{\alpha}\left(U_{f}^{\alpha}\right)^{*}\right)$ where $f \in \mathcal{L}^{2}(G), d_{\alpha}$ is the dimension of the irreducible representation indexed by $\alpha$, and $U_{f}=\int U_{x^{-1}} f(x) d x$. In the general case it is natural to deal with factor representations and to replace the trace by the Murray-von Neumann relative trace.$^{20} \mathrm{In}$ short, one is led to make the following conjecture. Let $S$ be a measure space effecting the decomposition of the right regular representation of $G$ into factor components. Then it is possible to choose a particular relative trace function $\mathrm{Tr}$ in the factor associated with each point $y$ of $S$ and a particular measure $\mu$ among

${ }^{19}$ Godement implicitly announces this result in a footnote in [28].

${ }^{20}$ For suitably restricted operators in any factor there is defined [57] a linear functional which has the formal properties of a trace. This function is unique up to multiplication by a positive real number. 
those with the given null sets in $S$ in such a way that for all $f \in \mathcal{L}^{2}$ $(G) \cap \mathcal{L}^{1}(G)$ the formula

$$
\int|f(x)|^{2} d x=\int \operatorname{Tr}\left(U_{f}^{y}\left(U_{f}^{y}\right)^{*}\right) d \mu(y)
$$

is valid. Such a formula has been established by F. I. Mautner [49] for discrete groups, by I. Kaplansky (unpublished) for CCR groups, by Gelfand and Neumark $[15 ; 18]$ for the group of all $n \times n$ complex matrices of determinant one, and by R. Godement $[24 ; 25]$ for central $^{21}$ groups. ${ }^{22}$ Very recently I. E. Segal [72] has been able to take care of the general case.

For a really thorough going generalization of the Plancherel theorem one would demand a little more; namely (a) an "onto" theorem asserting that the mapping $f \rightarrow U_{f}$ can be extended by continuity so as to map $\mathcal{L}^{2}(G)$ onto all functions $y \rightarrow A_{y}$ from $S$ to operators in the relevant factors which are measurable in a suitable sense and such that $\int \operatorname{Tr}\left[\left(A_{y}\left(A_{y}\right)^{*}\right] d(y)<\infty\right.$, (b) an a priori dual object; that is, a topological space of factor representations given in advance and while not capable necessarily of decomposing arbitrary representations, at least adequate for decomposing the right regular representation, (c) some sort of a generalization of Haar measure for the measure $\mu$; that is, a theorem asserting that $\mu$ can be chosen to have some invariance property with respect to Kronecker products of representations in the dual object and is uniquely defined by this property. The a priori dual object is at present available for central groups, CCR groups, and the group of all $n \times n$ complex matrices of determinant one. There is an onto theorem for the two-by-two case of the latter group. So far as the author is aware the invariant measure question has not been discussed for any non Abelian groups.

Just as for compact and Abelian groups, the Plancherel formula is connected with an expansion formula. ${ }^{23}$ For suitably restricted $f$ in $\mathcal{L}^{2}(G)$

$$
f(x)=\int \operatorname{Tr}\left(U_{x}^{y} U_{f}^{y}\right) d \mu(y)
$$

for all $x$ in $G$. This formula expresses $f$ as an integral with respect to $y$ of a family of functions, the " $y$ th" of which is a limit of finite linear

${ }^{21}$ Some of the statements in [24] are incorrect but have been corrected in a letter to I. Kaplansky. Presumably a corrected version of [24] will be published in due course.

${ }^{22}$ A central group is one whose group of inner autmorphisms has a compact closure in the suitably topologized group of all automorphisms. This notion is due to Godement.

${ }^{23}$ Cf. Lewitan [40]. 
combinations of "matrix elements" of the " $y$ th" factor representation. For Abelian groups the expansion formula not only sets up a one-toone correspondence between functions in $\mathcal{L}^{2}(G)$ and functions in $\mathcal{L}^{2}\left(G^{\wedge}\right)$, but also sets up such a correspondence between the positive definite functions on $G$ and the finite Borel measures on $G$. Specifically the formula $f(x)=\int(x, y) g(y) d y$ where $g \in \mathcal{L}^{1}(G)$ and $g(y) \geqq 0$ for all $y$ may be generalized by substituting a finite Borel measure $\alpha$ for $g$ and writing $f(x)=\int(x, y) d \alpha(y)$. The theorem that this formula sets up a one-to-one correspondence between measures and positive definite functions was proved by Herglotz when $G$ is the group of integers, by Bochner [5] when $G$ is the real line, and by Weil [78] and Raikov $[64 ; 65]$ for locally compact Abelian groups in general. The reader will find the close connection between this theorem and the Plancherel theorem discussed from various points of view in these papers and in a recent work of Cartan and Godement [7]. The paper of Cartan and Godement presents an elegant and concise exposition of a large part of the theory of locally compact Abelian groups starting with a simple derivation of the generalized HerglotzBochner theorem from the Krein-Milman theorem.

Because of the connection between positive definite functions and representations one can expect a close connection between expansion theorems for positive definite functions and decomposition theorems for unitary representations. For locally compact Abelian groups, for example, it is easy to deduce the Ambrose-Godement-Neumark generalization of Stone's theorem from the generalized Bochner-Herglotz theorem and vice versa. For non Abelian groups, on the other hand, the lack of a satisfactory dual object for decomposing general representations is reflected in the lack of a satisfactory generalization of the Bochner-Herglotz theorem. One can, however, guess at the form such a theorem would take; namely: If $G$ is a group with a dual object $S$ and $f$ is a continuous positive definite function on $G$, then there exists a measure $\alpha$ on $S$ and a function $A\left(y \rightarrow A_{y}\right)$ from $S$ to positive definite operators such that $A_{y}$ lies in a factor associated with $y$ and $f(x)=\int \operatorname{Tr}\left(U_{x}^{y} A_{y}\right) d \mu(y)$ where $U^{y}\left(x \rightarrow U_{x}^{\nu}\right)$ is the corresponding factor representation.

13. One-sided invariant subspaces. Very little has been done toward studying the one-sided invariant subspaces of $\mathcal{L}^{2}(G)$. Possibly the generalized direct sum theory can be applied to prove that each right invariant closed subspace of $\mathcal{L}^{2}(G)$ is defined by a suitably defined "measurable family" of right invariant closed subspaces-one at each point of the measure space effecting the decomposition of the 
right regular representation into factor components. If so, one is reduced to studying the right invariant subspaces in a Hilbert space under the action of a ring of operators which is a factor. The structure of such a family of subspaces is clear when the factor is of type $I_{n}$ or $I_{\infty}$. It is then order isomorphic to the family of all closed subspaces of a Hilbert space. For factors of other types it is not understood at all. W. Ambrose [2] has inaugurated a program which may throw some light on the situation. He proposes to regard $\mathcal{L}^{2}(G)$ as an "algebra" with a partially defined multiplication and to study its structure from the point of view of the Wedderburn theory. The left and right "ideals" in this algebra are the left and right closed invariant subspaces of $\mathcal{L}^{2}(G)$.

14. $\mathcal{L}^{1}(G)$ and other group algebras. There is also little that can be said about the ideal theory of $\mathcal{L}^{1}(G)$ when $G$ is neither compact nor Abelian. Such results as are available are primarily of a negative character. The representations of $\mathcal{L}^{1}(G)$ by bounded operators in Hilbert space, for example, are only loosely related to the ideal theory of $\mathcal{L}^{1}(G)$. Many non unitary equivalent such representations may have a common kernel ideal. Moreover it is possible for an irreducible representation to have a kernel ideal which is not maximal-there is an example in which such an ideal is the null ideal and at the same time an intersection of maximal ideals with finite-dimensional quotients. Perhaps the most significant positive result is that of $\mathrm{I}$. E. Segal [69] asserting that in general the zero ideal is an intersection of closed primitive ideals.

There are other ways, however, of generalizing the classical notion of group algebra and some of these lead to objects with a somewhat more tractable structure theory. I. E. Segal [70], for example, starts with the ring of all bounded linear operators in $\mathcal{L}^{2}(G)$ defined by convolution on the left with members of $\mathcal{L}^{1}(G)$ and proceeds to its uniform closure. A closely related algebra has been considered by Gelfand and Neumark [19]. Both of these algebras are isomorphic to uniformly closed algebras of operators in Hilbert space and such algebras (called $C^{*}$ algebras) have given rise to a rapidly growing literature. The connection between a group and its attached $C^{*}$ algebra is close enough so that many results about the one can be interpreted as results about the other, and vice versa, and in some cases the approach via the algebra has certain advantages. In particular the results of Kaplansky on CCR groups have been obtained in this way.

15. The determination of representations. In understanding the 
phenomena which arise in generalizing the Plancherel theorem, as well as for various other reasons, it is useful to have a more or less complete analysis of the irreducible and factor representations of particular groups which are neither compact nor Abelian. Such an analysis has been carried out by Bargmann [3] for the three- and four-dimensional Lorentz groups ${ }^{24}$ and by Gelfand and Neumark $[13 ; 14 ; 15 ; 16 ; 17]$ for the group of $n \times n$ complex matrices of determinant on $^{25}$ and the group of affine transformations of the line [12]. The author, as has already been indicated, has studied the representations of semi direct products of Abelian groups. The complete analysis which is possible in certain cases has led to a theorem [47] about "imprimitive" representations of arbitrary separable locally compact groups which promises to be useful in studying representations in general. In particular it turns out that every decomposition of the regular representation of a subgroup $G_{0}$ of a separable locally compact group $G$ as a generalized direct sum defines in a natural way a similar decomposition of the regular representation of $G$. This phenomenon when $G_{0}$ is Abelian has been discussed by Godement [28].

\section{APPENDIX}

(Added June 1950.) Since this article was written a number of further developments in the theory it describes have come to our attention. We sketch some of these below.

(1) Godement $[30 ; \mathbf{3 1} ; \mathbf{3 2} ; \mathbf{3 3}]$ has extended his theory of central groups to the more extensive family of groups of finite class. The definition of this notion is such that a group is of finite class if and only if every neighborhood of the identity admits a subneighborhood which is invariant under inner automorphisms. All discrete, compact, and locally compact Abelian groups are of finite class. Via a theory of "characters" for these groups Godement obtains a Plancherel theorem for them based on a topological a priori dual object. His work is closely related to and in part dependent on that of J. Dixmier [8] on rings of finite class.

(2) In a continuation of [49] Mautner has obtained independently of Segal a proof of the "weak" form of the general Plancherel theorem (\$12, paragraph 2 of this paper). Mautner's proof leans heavily on von Neumann's theory of weight functions for operator rings. Among other results in Mautner's paper is the theorem that if a factor repre-

${ }^{24}$ Cf. also Harish-Chandra [35].

${ }^{25}$ The quotient of the $2 \times 2$ group by its center is the four-dimensonal Lorentz group. 
sentation is not of type $I$, then any decomposition into irreducible parts has noncountably many components no two of which are unitary equivalent.

(3) Via a notion of dual semi-groups in a group $G$ and its "real character group" $\bar{G}$ the author has found a natural way of extending portions of the classical theory of the one-sided Laplace transform and of power series in a manner paralleling the generalization of the "two-sided" theory described in $\$ 8$. In particular one can prove a theorem about "completely monotonic" functions in semi-groups which generalizes the solution of the Hausdorff moment problem and the Hausdorff-Bernstein-Widder theorem on completely monotonic functions. The proof makes use of the Krein-Milman theorem in much the same way that Cartan and Godement used it to prove the Plancherel theorem.

\section{BIBLIOGRAPHY}

1. W. Ambrose, Spectral resolution of groups of unitary operators, Duke Math. J. vol. 11 (1944) pp. 589-595.

2. - The $L_{2}$ system of a unimodular group, Trans. Amer. Math. Soc. vol. 65 (1949) pp. 27-48.

3. V. Bargmann, Irreducible unitary representations of the Lorentz group, Ann. of Math. vol. 48 (1947) pp. 568-640.

4. S. Bochner, Beiträge zur Theorie der fastperiodischen Funktionen, Math. Ann. vol. 96 (1927) pp. 119-147. 1932.

5. ——, Vorlesungen iiber Fouriersche Integrale, Leipzig, Akad. Verlagsges.,

6. S. Bochner and J. von Neumann, Almost periodic functions in groups, Trans. Amer. Math. Soc. vol. 37 (1935) pp. 21-50.

7. H. Cartan and R. Godement, Théorie de la dualité et analyse harmonique dans les groupes abéliens localement compact, Ann. École Norm. vol. 64 (1947) pp. 79-99.

8. J. Dixmier, Les anneaux d'operateurs de classe finie, Ann. Ecole Norm. (3) vol. 66 (1950) pp. 209-261.

9. H. Freudenthal, Topologische Gruppen mit genugend vielen fastperiodischen Funktionen, Ann. of Math. vol. 37 (1936) pp. 57-77.

10. I. Gelfand, Normierte Ringe, Rec. Math. (Mat. Sbornik) N. S. vol. 9 (1941) pp. 3-24.

11. - Über absolut konvergente trigonometrische Reihen und Integrale, Rec. Math. (Mat. Sbornik) N. S. vol. 9 (1941) pp. 51-66.

12. I. Gelfand and M. Neumark, Unitary representations of the group of linear transformations of the straight line, C. R. (Doklady) Acad. Sci. URSS. vol. 55 (1947) pp. 567-570.

13. - The principal series of irreducible representations of the complex unimodular group, C. R. (Doklady) Acad. Sci. URSS. vol. 56 (1947) pp. 3-4.

14. - Supplementary and degenerate series of representations of the complex unimodular group, C. R. (Doklady) Acad. Sci. URSS. vol. 58 (1947) pp. 1577-1580.

15. - Unitary representations of the Lorentz group, Izvestiya Akademii Nauk SSSR. Ser. Mat. vol. 11 (1947) pp. 411-504. 
16. - Unitary representations of semi simple Lie groups I, Rec. Math. (Mat. Sbornik) N. S. vol. 21 (1947) pp. 405-434.

17. - On the connection between the representations of a complex simple Lie group and those of its maximal compact subgroups, Doklady Akad. Nauk SSSR. vol. 63 (1948) pp. 225-228.

18. - The analogue of Plancherel's formula for the complex unimodular group, Doklady Akad. Nauk SSSR. vol. 63 (1948) pp. 609-612.

19. - Normed rings with involution and their representations, Izvestiya Akademii Nauk SSSR. Ser. Mat. vol. 12 (1948) pp. 445-480.

20. I. Gelfand and D. Raikov, On the theory of character of commutative topological groups, C. R. (Doklady) Acad. Sci. URSS. vol. 28 (1940) pp. 195-198.

21. - Irreducible unitary representations of locally bicompact groups, Rec. Math. (Mat. Sbornik) N. S. vol. 13 (1943) pp. 301-316.

22. I. Gelfand, D. Raikov, and G. Silov, Commutative normed rings, Uspehi Matematičeskih Nauk. N. S. vol. 2 (1946) pp. 48-146.

23. R. Godement, Sur une généralisation d'un théorème de Stone, C. R. Acad. Sci. Paris vol. 218 (1944) pp. 901-903.

24. - Analyse harmonique dans les groupes centraux I. Fonctions centrales et caracteres, C. R. Acad. Sci. Paris vol. 225 (1947) pp. 19-21.

25. - Analyse harmonique dans les groupes centraux II. Formule d'inversion de Fourier, C. R. Acad. Sci. Paris vol. 225 (1947) pp. 221-223.

26. - Thêorèmes taubériens et théorie spectrale, Ann. École Norm. vol. 64 (1947) pp. 119-138.

27. - Les fonctions de type positif et la thêorie des groupes, Trans. Amer. Math. Soc. vol. 63 (1948) pp. 1-84.

28. - Sur la transformation de Fourier dans les groupes discrets, C. R. Acad. Sci. Paris vol. 228 (1949) pp. 627-628.

29. - Théorie générale des sommes continues d'espaces de Banach, C. R. Acad. Sci. Paris vol. 228 (1949) pp. 1321-1323.

30. - Sur la thêorie des caractères. I. Definition et classification des caractères, C. R. Acad. Sci. Paris vol. 229 (1949) pp. 967-969.

31. - Sur la théorie des caractères. II. Mesures et groupes de classe finie, C. R. Acad. Sci. Paris vol. 229 (1949) pp. 1050-1051.

32. - Sur la théorie des caractères. III. Un exemple de mesure-caractère de classe (I), C. R. Acad. Sci. Paris vol. 229 (1949) pp. 1107-1109.

33. - Mémoire sur la thêorie des caractères dans les groupes localement compacts unimodulaire, to be published.

34. A. Haar, Der Massbegriff in der Theorie der kontinuierlichen Gruppen, Ann. of Math. vol. 34 (1933) pp. 147-169.

35. Harish-Chandra, Infinite irreducible representations of the Lorentz group, Proc. Royal Soc. London Ser. A vol. 189 (1947) pp. 372-401.

36. P. R. Halmos, Measure theory, New York, 1950.

37. A. Hurevitsch, Unitary representations in Hilbert space of a compact topological group, Rec. Math. (Mat. Sbornik) N. S. vol. 13 (1943) pp. 79-86.

38. S. Kakutani, On the uniqueness of Haar's measure, Proc. Imp. Acad. Tokyo vol. 14 (1938) pp. 27-31.

39. E. R. van Kampen, Locally bicompact groups and their character groups, Ann. of Math. vol. 36 (1935) pp. 448-463.

40. - Almost periodic functions and compact groups, Ann. of Math. vol. 37 (1936) pp. 78-91. 
41. I. Kaplansky, Primary ideals in group algebras, Proc. Nat. Acad. Sci. U.S.A. vol. 35 (1949) pp. 133-136.

42. G. Köthe, Abstracte Theorie nichtkommutativer Ringe mit einer Anwendung auf die Darstellungstheorie kontinuierlicher Gruppen, Math. Ann. vol. 103 (1930) pp. 545572.

43. M. Krein, Sur une généralisation du théorème de Plancherel au cas des integrales de Fourier sur les groupes topologiques commutatifs. C. R. (Doklady) Acad. Sci. URSS. vol. 30 (1941) pp. 484-488.

44. M. Krein and D. Milman, On extreme points of regular convex sets, Studia Mathematica vol. 9 (1940) pp. 133-138.

45. B. Lewitan, On the theory of unitary representations of locally compact groups, Rec. Math. (Mat. Sbornik) N. S. vol. 19 (1946) pp. 407-427.

46. G. W. Mackey, The Laplace transform for locally compact Abelian groups, Proc. Nat. Acad. Sci. U.S.A. vol. 34 (1948) pp. 156-162.

47. - Imprimitivity for representations of locally compact groups, Proc. Nat. Acad. Sci. U.S.A. vol. 35 (1949) pp. 537-545.

48. F. I. Mautner, The completeness of the irreducible unitary representations of a locally compact group, Proc. Nat. Acad. Sci. U.S.A. vol. 34 (1948) pp. 52-54.

49. - Unitary representations of locally compact groups I, Ann. of Math. vol. 51 (1950) pp. 1-25.

50. - Completeness relations on locally compact groups, Bull. Amer. Math. Soc. Abstract 55-1-46.

51. - Unitary representations of locally compact groups II, Ann. of Math. to be published.

52. F. J. Murray and J. von Neumann, On rings of operators, Ann. of Math. vol. 37 (1935) pp. 116-229.

53. - On rings of operators IV, Ann. of Math. vol. 44 (1943) pp. 716-808.

54. J. von Neumann, Almost periodic functions in a group, Trans. Amer. Math. Soc. vol. 36 (1934) pp. 445-492.

55. - The uniqueness of Haar's measure, Rec. Math. (Mat. Sbornik) N. S. vol. 1 (1936) pp. 721-734.

56. - Invariant measures, Lecture Notes, Institute for Advanced Study, Princeton, 1941.

57. - On rings of operators III, Ann. of Math. vol. 41 (1940) pp. 94-161.

58. - On rings of operators. Reduction theory, Ann. of Math. vol. 50 (1949) pp. 401-485.

59. M. Neumark, Positive definite operator functions on a commutative group, Izvestiya Akademii Nauk SSSR. Ser. Math. vol. 7 (1943) pp. 237-244.

60. F. Peter and H. Weyl, Die Vollständigkeit der primitiven Darstellungen einer geschlossene kontinuierlichen Gruppe, Math. Ann. vol. 97 (1927) pp. 737-755.

61. L. Pontrjagin, Les fonctions presque periodiques et l'analysis situs, C. R. Acad. Sci. Paris vol. 196 (1933) pp. 1201-1203.

62. - The theory of topological commutative groups, Ann. of Math. vol. 35 (1934) pp. 361-388.

63. - Topological groups, Princeton, 1939.

64. D. Raikov, Positive definite functions on commutative groups with an invariant measure, C. R. (Doklady) Acad. Sci. URSS. vol. 28 (1940) pp. 296-300.

65. - Harmonic analysis on commutative groups with the Haar measure and the theory of characters, Travaux de l'Institut Mathématique Stekloff vol. 14 (1945).

66. L. Schwartz, Théorie générale des fonctions moyenne-periodiques, Ann. of Math. vol. 48 (1947) pp. 857-929. 
67. - Sur une propriêté de synthèse spectrale dans les groupes non compacts, C. R. Acad. Sci. Paris vol. 227 (1948) pp. 424-426.

68. I. E. Segal, The group ring of a locally compact group I, Proc. Nat. Acad. Sci. U. S. A. vol. 27 (1941) pp. 348-352.

69. - The group algebra of a locally compact group, Trans. Amer. Math. Soc. vol. 61 (1947) pp. 69-105.

70. - Irreducible representations of operator algebras, Bull. Amer. Math. Soc. vol. 53 (1947) pp. 73-88.

71. - The two sided regular representation of a unimodular locally compact group, Ann. of Math. vol. 51 (1950) pp. 293-298.

72. - A generalization of the Plancherel formula for separable unimodular locally compact groups, to be published.

73. M. H. Stone, On one parameter unitary groups in Hilbert space, Ann. of Math. vol. 33 (1932) pp. 643-648.

74. S. Tannaka, Dualität der nicht-kommutativen Gruppen, Tôhoku Math. J. vol. 53 (1938) pp. 1-12.

75. G. Thorin, An extension of a convexity theorem due to M. Riesz, Kungl. Fysiografiska Sällskopets i Lund Fördhandlingar vol. 8 (1939) no. 14.

76. A. Weil, Sur les fonctions presque periodique de von Neumann, C. R. Acad. Sci. Paris vol. 200 (1935) pp. 38-40.

77. - Sur les groupes topologiques et les groupes mesures, C. R. Acad. Sci. Paris vol. 202 (1936) pp. 1147-1149.

78. —- L'intégration dans les groupe topologiques et ses applications, Actualités Scientifique et Industrielles, no. 869, Paris, 1938.

79. H. Weyl, Harmonics on homogeneous manifolds, Ann. of Math. vol. 35 (1934) pp. $486-499$.

80. N. Wiener, The Fourier integral and certain of its applications, Cambridge University Press, 1933.

81. E. P. Wigner, On unitary representations of the inhomogeneous Lorentz group, Ann. of Math. vol. 40 (1939) pp. 149-204.

\section{HaRvard UNIVERSITY}

